\title{
THE MAXIMAL PRIME GAPS SUPREMUM AND THE FIROOZBAKHT'S HYPOTHESIS $\mathrm{N}^{O} 30$
}

\author{
JAN FELIKSIAK
}

\begin{abstract}
The maximal prime gaps upper bound problem is one of the major mathematical problems to date. The objective of current research is to develop a standard which will aid in the understanding of the distribution of prime numbers. This paper presents theoretical results which originated with a research in the subject of the maximal prime gaps. The document presents the sharpest upper bound for the maximal prime gaps ever developed. The result becomes the Supremum bound on the maximal prime gaps and subsequently culminates with the conclusive proof of the Firoozbakht's Hypothesis $N^{\circ} 30$. Firoozbakht Hypothesis implies quite a bold conjecture concerning the maximal prime gaps. In fact it imposes one of the strongest maximal prime gaps bounds ever conjectured. Its truth implies the truth of a greater number of known prime gaps conjectures, simultaneously the Firoozbakht's Hypothesis disproves a known heuristic argument of Granville and Maier. This paper is dedicated to a fellow mathematician, the late Farideh Firoozbakht.
\end{abstract}

(C)2014 Jan Feliksiak

2000 Mathematics Subject Classification. 0102, 11A41, 11K65, 11L20, 11N05, 1102, 1103.

Key words and phrases. Cramér's conjecture, Elementary proof, Firoozbakht Conjecture, Farideh Firoozbakht, Legendre Conjecture, Maximal prime gaps Supremum, Prime gaps. 


\section{The BINOmial EXPANSION $2^{\left(n+\mathcal{G} s_{(n)}\right)}$}

\subsection{Preliminaries.}

Within the scope of the paper, prime gap of the size $\mathfrak{g} \in \mathbb{N} \mid \mathfrak{g} \geq 2$ is defined as an interval between two primes $\left(p_{i}, p_{i+1}\right]$, containing $(\mathfrak{g}-1)$ composite integers. Maximal prime gap of the size $\mathfrak{g}$, is a gap strictly exceeding in size any preceding gap. All calculations and graphing were carried out with the aid of the Mathematica ${ }^{\circledR}$ software. For all $n \in \mathbb{N} \mid n \geq 8$, we make the following definitions:

Definition 1.1 (Interval length). $c=\mathcal{G} s_{(n)}=\left\lfloor 5\left(\log _{10} n\right)^{2}-\frac{15}{8}\left(\log _{10} n\right)\right\rfloor$

Definition 1.2 (Interval endpoint). $t=(n+c)$

The binomial coefficient $\mathcal{M}_{(t)}$ related to the current research is a part of the associated binomial expansion:

$$
2^{t}>>\left(\begin{array}{c}
n+c \\
n
\end{array}\right)
$$

Definition 1.3 (Binomial coefficient).

$$
\mathcal{M}_{(t)}=\left(\begin{array}{c}
n+c \\
n
\end{array}\right)=\left(\frac{(n+c) !}{(n ! \times c !)}\right)
$$

Definition 1.4 (Logarithm of the binomial coefficient).

$\log \mathcal{M}_{(t)}=\log \left(\frac{(n+c) !}{(n ! \times c !)}\right)=\log (t !)-\log (n !)-\log (c !)=\sum_{k=1}^{c} \log (n+k)-\sum_{k=1}^{c} \log k$

Graphs in this paper implement a variant of logarithmic scaling of the horizontal axis given by:

Definition 1.5 (Scaling factor). $\xi=\frac{\log _{10}\left(\frac{n}{24}\right)}{\log _{10}(24)}$

\section{Remark 1.1.}

The function $\mathcal{G} s_{(n)}$ is an increasing, weakly monotone function. Due to the floor function used, $\mathcal{G} s_{(n)}$ clearly increases stepwise, producing a sequence in $\mathbb{N}$.

The logarithm of the binomial coefficient $\log \mathcal{M}_{(t)}$ produces an increasing, strictly monotone sequence in $\mathbb{R}$. The sudden increase in value of the function $\mathcal{G} s_{(n)}$, causes an analogous simultaneous jump in value of the associated binomial coefficient $\mathcal{M}_{(t)}$. Because at all other intermediate points of that particular interval the binomial coefficient $\mathcal{M}_{(t)}$ increases steadily, therefore the graph of $\log \mathcal{M}_{(t)}$ forms a distinctive slanted staircase pattern. 


\subsection{Bounds on the logarithm of the binomial coefficient.}

Lemma 1.6 (Upper and Lower bounds on the logarithm of $n !$ ).

The bounds on the logarithm of $n$ ! are given by:

$$
n \log (n)-n+1 \leq \log (n !) \leq(n+1) \log (n+1)-n \quad \forall n \in \mathbb{N} \mid n \geq 5
$$

Proof.

Evidently,

$$
\log (n !)=\sum_{k=1}^{n} \log (k) \quad \forall n \in \mathbb{N} \mid n \geq 2
$$

Hence, the pertinent integrals to consider are:

$$
\int_{1}^{n} \log (x) d x \leq \log (n !) \leq \int_{0}^{n} \log (x+1) d x \quad \forall n \in \mathbb{N} \mid n \geq 5
$$

Accordingly, evaluating those integrals we obtain:

$$
\begin{aligned}
n \log (n)-n+1 \leq \log (n !) \leq n \log \left(\frac{(n+1)}{e}\right)+ & \log \left(\frac{(n+1)}{e}\right)+1 \\
& =(n+1) \log (n+1)-n
\end{aligned}
$$

Concluding the proof of Lemma 1.6.

\section{Remark 1.2.}

Observe that $\log \mathcal{M}_{(t)}$ is a difference of logarithms of factorial terms:

$$
\log \mathcal{M}_{(t)}=(\log (t !)-\log (n !)-\log (c !))
$$

Consequently, implementing the lower/upper bounds on the logarithm of $n$ ! for the bounds on $\log \mathcal{M}_{(t)}$, results in bounds of the form:

$$
\log \left(\frac{(t+k)^{(t+k)}}{(n+k)^{(n+k)}(c+k)^{(c+k)}}\right) \quad \text { for } \forall k \in \mathbb{N} \cup\{0\}
$$

Keeping the values of $c, n$ and $t$ constant and letting the variable $k$ to increase unboundedly, results in an unboundedly decreasing function. When implementing the lower/upper bounds on the logarithm of $n$ ! for the Supremum/Infimum bounds on $\log \mathcal{M}_{(t)}$, the variable $k$ appears only with values $k=\{0,1\}$ respectively. The combined effect of the difference of the logarithms of factorial terms in $\log \mathcal{M}_{(t)}$ and the decreasing property of the function 1.5, imposes a reciprocal interchange of the bounds 1.1, when implementing them for the bounds on $\log \mathcal{M}_{(t)}$.

Lemma $1.7\left(\log \mathcal{M}_{(t)}\right.$ Supremum Bound). by:

The Supremum Bound on the logarithm of the binomial coefficient $\mathcal{M}_{(t)}$ is given

$$
\log \mathcal{M}_{(t)} \leq \log \left(\frac{t^{t}}{n^{n} c^{c}}\right)-1=\mathcal{U} \mathcal{B}_{(t)} \quad \forall n \in \mathbb{N} \mid n \geq 8
$$


Proof.

From Lemma1.6 we have:

$$
(n \log (n)-n+1) \leq \log (n !)
$$

Substituting from the inequality 1.7 into the Definition 1.4 we obtain:

$$
\begin{aligned}
& (\log (t !)-\log (n !)-\log (c !)) \\
& \leq((t \log (t)-t+1)-(n \log (n)-n+1)-(c \log (c)-c+1)) \\
& =t \log (t)-n \log (n)-c \log (c)-1=\log \left(\frac{t^{t}}{n^{n} c^{c}}\right)-1
\end{aligned}
$$

Consequently,

$$
\log \mathcal{M}_{(t)} \leq \log \left(\frac{t^{t}}{n^{n} c^{c}}\right)-1=\mathcal{U} \mathcal{B}_{(t)}
$$

The Supremum bound $\mathcal{U} \mathcal{B}_{(t)}$ produces an increasing, strictly monotone sequence in $\mathbb{R}$. At $n=8$, the difference $\mathcal{U} \mathcal{B}_{(t)}-\log \mathcal{M}_{(t)}$ attains 0.197362 and diverges as $n \rightarrow \infty$. Therefore, Lemma 1.7 holds as specified.

Lemma $1.8\left(\log \mathcal{M}_{(t)}\right.$ Infimum Bound).

The Infimum Bound on the natural logarithm of the binomial coefficient $\mathcal{M}_{(t)}$ for all $n \in \mathbb{N} \mid n \geq 8$ is given by:

$$
\log \mathcal{M}_{(t)} \geq \log \left(\frac{(t+1)^{(t+1)}}{(n+1)^{(n+1)}(c+1)^{(c+1)}}\right)=\mathcal{L B}_{(t)}
$$

Proof.

From Lemma1.6 we have:

$$
\log (n !) \leq n \log (n+1)-n+\log (n+1)
$$

Substituting from the inequality 1.11 into the Definition 1.4 we obtain:

$$
(\log (t !)-\log (n !)-\log (c !))
$$

$$
\geq t \log (t+1)-n \log (n+1)-c \log (c+1)+\log (t+1)-\log (n+1)-\log (c+1)
$$

$$
=\log \left(\frac{(t+1)^{t}}{(n+1)^{n}(c+1)^{c}}\right)+\log \left(\frac{(t+1)}{(n+1)(c+1)}\right)
$$

Consequently,

$$
\log \mathcal{M}_{(t)} \geq \log \left(\frac{(t+1)^{(t+1)}}{(n+1)^{(n+1)}(c+1)^{(c+1)}}\right)=\mathcal{L B}_{(t)}
$$

The Infimum Bound $\mathcal{L B}_{(t)}$ produces an increasing, strictly monotone sequence in $\mathbb{R}$. At $n=8$, the difference $\log \mathcal{M}_{(t)}-\mathcal{L B}_{(t)}$ attains 0.500673 and diverges as $n \rightarrow \infty$. Therefore, Lemma 1.8 holds as specified.

Consequently, from Lemma 1.8 and 1.7 we have for all $n \in \mathbb{N} \mid n \geq 8$ :

$$
\log \left(\frac{(t+1)^{(t+1)}}{(n+1)^{(n+1)}(c+1)^{(c+1)}}\right) \leq \log \mathcal{M}_{(t)} \leq \log \left(\frac{t^{t}}{n^{n} c^{c}}\right)-1
$$



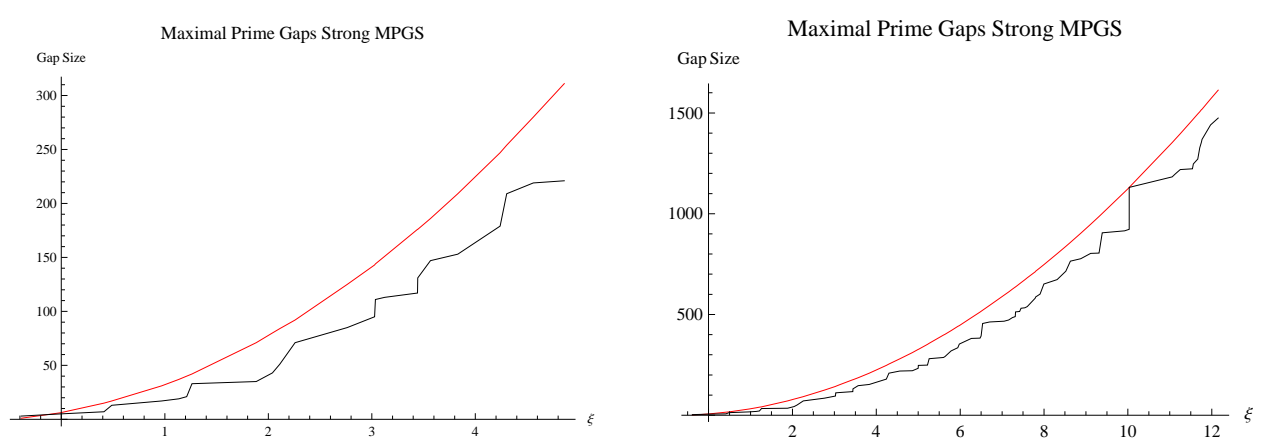

FiguRE 1. shows the graphs of $\mathcal{G} s_{(n)}$ (red) and the actual maximal gaps (black) with respect to $\xi$ as given by Def. 1.5. The graph has been produced on the basis of data obtained from $\mathrm{C}$. Caldwell as well as from T. Nicely tables of maximal prime gaps. The occurrence of the gap $64 ; \mathfrak{g}=1131$, beginning at the prime 1693182318746371 , is abnormally low (T. Oliveira e Silva, 2006). It is clearly visible on the graph at $\xi \approx 10.03$ with the difference $\mathcal{G} s_{(n)}-\mathfrak{g}=0.0132662$.

\section{MAXimal PRIME Gaps}

From the Prime Number Theorem we have that an average gap between consecutive primes is given by $\log n$ for any $n \in \mathbb{N}$. There exist however prime gaps much shorter - containing only a single composite number, and gaps which are much longer than average - the maximal prime gaps.

We begin with a preliminary derivation. Since the integers from 1 to $\mathrm{n}$ contain $\left\lfloor\frac{n}{p}\right\rfloor$ multiples of the prime number $\mathrm{p},\left\lfloor\frac{n}{p^{2}}\right\rfloor$ multiples of $p^{2}$ etc. Thus it follows that:

$$
n !=\prod_{p} p^{u_{(n, p)}} ; \text { where } u_{(n, p)}=\sum_{m \geq 1}\left\lfloor\frac{n}{p^{m}}\right\rfloor
$$

In accordance with the definitions 1.1 of $\mathcal{G}_{s_{(n)}}, 1.2$ of $t$ and 1.3 of $\mathcal{M}_{(t)}$ we obtain:

$$
\mathcal{M}_{(t)}=\prod_{p \leq t} p^{\mathcal{K}_{p}}
$$

where

$$
\mathcal{K}_{p}=\sum_{m=1}^{\infty}\left(\left\lfloor\frac{t}{p^{m}}\right\rfloor-\left\lfloor\frac{n}{p^{m}}\right\rfloor-\left\lfloor\frac{\mathcal{G} s_{(n)}}{p^{m}}\right\rfloor\right)
$$

it follows that

$$
\mathcal{K}_{p} \leq\left\lfloor\frac{\log t}{\log p}\right\rfloor
$$

and so by the above, Lemma 1.7 and 1.8 we have:

$$
\mathcal{L B}_{(t)} \leq \log \mathcal{M}_{(t)}=\log \prod_{p \leq t} p^{\mathcal{K}_{p}}=\sum_{p \leq t} \mathcal{K}_{p} \log p \leq \mathcal{U} \mathcal{B}_{(t)} \quad \forall n \in \mathbb{N} \mid n \geq 8
$$

Where $\mathrm{p}$ is as usual a prime number. Let's define: 
Definition 2.1. $s=\left\lfloor\frac{\left(n+\mathcal{G} s_{(n)}\right)}{2}\right\rfloor=\left\lfloor\frac{t}{2}\right\rfloor$

Lemma 2.2 (Prime Factors of $\mathcal{M}_{(t)}$ ).

The case when there does not exist any prime factor $p$ of $\mathcal{M}_{(t)}$ within the interval from $n$ to $\left(n+\mathcal{G} s_{(n)}\right)=t$ for any $n \in \mathbb{N} \mid n \geq 11$, imposes an upper limit on all prime factors $p$ of $\mathcal{M}_{(t)}$. Consequently in this particular case, every prime factor $p$ must be less than or equal to $s=\left\lfloor\frac{t}{2}\right\rfloor$.

Proof.

Let $\mathrm{p}$ be a prime factor of $\mathcal{M}_{(t)}$ so that $\mathcal{K}_{p} \geq 1$ and suppose that every prime factor $p \leq n$. If

$$
s<p \leq n
$$

then,

and

$$
p<\left(n+\mathcal{G} s_{(n)}\right)<2 p
$$

$$
p^{2}>\left(\frac{\left(n+\mathcal{G} s_{(n)}\right)}{2}\right)^{2}>\left(n+\mathcal{G} s_{(n)}\right)
$$

and so $\mathcal{K}_{p}=0$. Therefore $p \leq s$ for every prime factor $\mathrm{p}$ of $\mathcal{M}_{(t)}$, for any $n \in \mathbb{N} \mid n \geq 11$.

2.1. Maximal Prime Gaps Supremum. The bounds on the logarithm of $\mathcal{M}_{(t)}$ are given by Lemma 1.7 and 1.8:

$$
\begin{aligned}
& \mathcal{L B}_{(t)}=\log \left(\frac{(t+1)^{(t+1)}}{(n+1)^{(n+1)}(c+1)^{(c+1)}}\right) \\
& \leq \log \mathcal{M}_{(t)}=\sum_{k=1}^{c} \log (n+k)-\sum_{k=1}^{c} \log k \leq \log \left(\frac{t^{t}}{n^{n} c^{c}}\right)-1=\mathcal{U B}_{(t)} \\
& \forall n \in \mathbb{N} \mid n \geq 11
\end{aligned}
$$

\section{Remark 2.1.}

- The proof of the Maximal Prime Gaps Supremum implements the Supremum bound function $\mathcal{U B}_{\left(t_{s}\right)}$. Due to the fact that the function $\mathcal{U} \mathcal{B}_{(t)}$ applies values of $n, c$ and $t$ directly, it imposes a requirement to generate a set of pertinent values to correctly approximate the interval $s$. This is to ascertain that the generated interval is at least equal or greater than $s$ as given by Definition 2.1, as well as the corresponding value of $c$. Respective definitions follow:

Definition 2.3. $n_{s}=\frac{n}{2}$

Definition 2.4. $c_{s}=5\left(\log _{10} n_{s}\right)^{2}-\frac{15}{8}\left(\log _{10} n_{s}\right)+1$

Definition 2.5. $t_{s}=n_{s}+c_{s}$

- The function $\mathcal{G} s_{(n)}$ due to the implementation of the Floor function increases stepwise. The sudden increase in value of the function $\mathcal{G}_{s_{(n)}}$ is mirrored by an analogous, simultaneous increase in both, implemented bounds on the function $\log \mathcal{M}_{(t)}$ as well as the function $\log \mathcal{M}_{(t)}$ itself. This is especially important for any kind of test or computation. The bottom peaks occur at $n-1$ immediately preceding the sudden increase of value of $c=\mathcal{G} s_{(n)}$ at $n$. 
Theorem 2.6 (Maximal Prime Gaps Supremum and Infimum for primes).

For any $n \in \mathbb{N} \mid n \geq 11$ there exists at least one $p \in \mathbb{N} \mid n<p \leq n+\mathcal{G} s_{(n)}=t$; where $p$ is as usual a prime number and the maximal prime gaps standard measure $\mathcal{G} s_{(n)}$ is given by:

$$
\begin{array}{r}
\mathcal{S U P}=\mathcal{G} s_{(n)}=\left\lfloor 5\left(\log _{10} n\right)^{2}-\frac{15}{8}\left(\log _{10} n\right)\right\rfloor \quad \forall n \in \mathbb{N} \mid n \geq 11 \\
\text { Equivalently, } p_{i+1}-p_{i} \leq \mathcal{G} s_{\left(p_{i}\right)}
\end{array}
$$

Proof.

Suppose that there is no prime within the interval from $n$ to $t$. Then in accordance with the hypothesis, by Lemma 2.2 we have that every prime factor $p$ of $\mathcal{M}_{(t)}$ must be less than or equal to $s=\left\lfloor\frac{t}{2}\right\rfloor$. Invoking Definitions 2.3, 2.4 and 2.5, Lemma $1.7,1.8$ and the inequality 2.1 we obtain in such a case, for all $n \in \mathbb{N} \mid n \geq 11$ :

$$
\begin{aligned}
& (2.4) \mathcal{L B}_{(t)}=\log \left(\frac{(t+1)^{(t+1)}}{(n+1)^{(n+1)}(c+1)^{(c+1)}}\right) \\
& \leq \log \mathcal{M}_{(t)}=\log \prod_{p \leq t_{(s)}} p^{\mathcal{K}_{p}}=\sum_{p \leq t_{(s)}} \mathcal{K}_{p} \log p \leq \log \left(\frac{\left(t_{s}\right)^{t_{s}}}{\left(n_{s}\right)^{n_{s}}\left(c_{s}\right)^{c_{s}}}\right)-1=\mathcal{U} \mathcal{B}_{\left(t_{s}\right)}
\end{aligned}
$$

In accordance with the hypothesis therefore, it must be true that:

$$
\log \left(\frac{(t+1)^{(t+1)}}{(n+1)^{(n+1)}(c+1)^{(c+1)}}\right)-\log \left(\frac{\left(t_{s}\right)^{t_{s}}}{\left(n_{s}\right)^{n_{s}}\left(c_{s}\right)^{c_{s}}}\right)+1 \leq 0
$$

However, at $n=47$ the difference 2.5 attains $\sim 7.69823$ and diverges as $n$ increases unboundedly. Since the difference generates a positive sequence in $\mathbb{R}$, we apply therefore the Cauchy's Root Test for $n \geq 47$ :

$$
\limsup _{c \rightarrow \infty} \sqrt[c]{\left|a_{c}\right|}=\limsup _{c \rightarrow \infty} \sqrt[c]{\mathcal{L B}_{(t)}-\mathcal{U} \mathcal{B}_{\left(t_{s}\right)}} \rightarrow 1
$$

At $n=47$ the Cauchy's Root Test attains $\approx 1.20947$ and tends asymptotically to 1 decreasing strictly from above. Thus, by the definition of the Cauchy's Root Test, the series formed from the terms of the difference $\mathcal{L} \mathcal{B}_{(t)}-\mathcal{U} \mathcal{B}_{\left(t_{s}\right)}$ diverges to infinity as $c$ increases unboundedly. This implies that for all $n \in \mathbb{N} \mid n \geq 47$ :

$$
\mathcal{L B}_{(t)}-\mathcal{U B}_{\left(t_{s}\right)}>0
$$

Hence, we have a contradiction to the initial hypothesis. Necessarily therefore, there must be at least one prime within the interval $c$ for all $n \in \mathbb{N} \mid n \geq 47$. Table 1 lists all values of $n$ s.t. $11 \leq n \leq 53$. Evidently, every possible sub-interval contains at least one prime number. Thus we deduce that Theorem 2.6 holds in this range as well. Consequently Theorem 2.6 holds as stated for all $n \in \mathbb{N} \mid n \geq 11$, thus completing the proof.

Remark 2.2. We may now slightly relax the function $\mathcal{G} s_{(n)}$ by dropping the Floor function, if needed. 
TABLE 1. Low range $\mathcal{G} s_{(n)}$ vs primes within the range

\begin{tabular}{|c|c|c|c|c|c|}
\hline$n$ & $\mathcal{G} s_{(n)}$ & primes & $n$ & $\mathcal{G} s_{(n)}$ & primes \\
\hline 11 & 3 & 13 & 31 & 8 & 37 \\
\hline 13 & 4 & 17 & 37 & 9 & 41,43 \\
\hline 17 & 5 & 19 & 41 & 9 & 43,47 \\
\hline 19 & 5 & 23 & 43 & 10 & 47,53 \\
\hline 23 & 6 & 29 & 47 & 10 & 53 \\
\hline 29 & 7 & 31 & 53 & 11 & 59,61 \\
\hline
\end{tabular}

\section{Firoozbakht's Hypothesis $\mathrm{N}^{\circ} 30$}

In 1982, an Iranian mathematician Farideh Firoozbakht formulated a conjecture:

Theorem 3.1 (Firoozbakht's Hypothesis $\mathrm{N}^{\circ} 30$ ).

The Firoozbakht Hypothesis defined by the relation:

$$
\sqrt[n]{p_{(n)}}>\sqrt[(n+1)]{p_{(n+1)}}
$$

is valid for all $p_{n} \in \mathbb{N} \mid p_{n} \geq 2$. Where $n \in \mathbb{N} \mid n \geq 1$ is the index of the $n$-th prime number.

Proof.

$$
\sqrt[n]{p_{(n)}}>\sqrt[(n+1)]{p_{(n+1)}} \equiv\left(p_{n}\right)^{(n+1)}>\left(p_{(n+1)}\right)^{n}
$$

Now, $\left(p_{n}\right)^{(n+1)}=\left(p_{n}\right)^{n} p_{n}$. Therefore, upon substitution into 3.2 we obtain:

$$
\left(p_{n}\right)^{n} p_{n}>\left(p_{(n+1)}\right)^{n} \equiv p_{n}>\left(\frac{p_{(n+1)}}{p_{n}}\right)^{n}
$$

Since the prime gap $\mathfrak{g}=p_{(n+1)}-p_{n}$ thus, $p_{(n+1)}=p_{n}+\mathfrak{g}$. Hence, from 3.3 we have:

$$
p_{n}>\left(\frac{p_{n}+\mathfrak{g}}{p_{n}}\right)^{n}
$$

Taking logs,

$$
\frac{\log p_{n}}{n}>\log \left(\frac{p_{n}+\mathfrak{g}}{p_{n}}\right) \equiv \frac{\log p_{n}}{n}>\log \left(1+\frac{\mathfrak{g}}{p_{n}}\right)
$$

By the Theorem 2.6, maximal prime gaps are bounded above by the Supremum bound:

$$
\begin{aligned}
& \mathfrak{g}=p_{(n+1)}-p_{n} \leq \mathcal{S U P}=5\left(\log _{10} p_{(n)}\right)^{2}-\frac{15}{8}\left(\log _{10} p_{(n)}\right) \\
& \forall p_{(n+1)} \in \mathbb{N} \mid p_{(n+1)} \geq 13
\end{aligned}
$$

Therefore, substituting $\mathcal{S U} \mathcal{P}$ for the prime gaps $\mathfrak{g}$ into 3.5 , we obtain:

$$
\frac{\log p_{n}}{n}>\log \left(1+\frac{\mathcal{S U P}}{p_{n}}\right)
$$

Clearly,

$$
\lim _{n \rightarrow \infty}\left(\log \left(1+\frac{\mathcal{S U \mathcal { P }}}{p_{n}}\right)\right) \rightarrow 0
$$


Let's examine in turn, the first term of 3.6. By the PNT we have that:

$$
n \log n+n(\log \log n-1)<p_{n}<n \log n+n \log \log n \quad \forall n \in \mathbb{N} \mid n \geq 6
$$

Hence, from the Inequality 3.8, we have that:

$$
p_{n}<n \log n+n \log \log n
$$

Clearly, both sides of the inequality 3.9 diverge as $n \rightarrow \infty$ therefore, we substitute RHS of 3.9 into the first term of 3.6 thereby obtaining:

$$
\frac{\log p_{n}}{n} \leq \frac{\log (n \log n+n \log \log n)}{n}=\frac{\log n+\log (\log n)+\log \left(1+\frac{\log \log n}{\log n}\right)}{n}
$$

Now, for any $n \in \mathbb{N} \mid n \geq 6$, the limit of 3.10 by the L'Hôpital's rule is:

$$
\begin{aligned}
\lim _{n \rightarrow \infty}\left(\frac{\log p_{n}}{n}\right)=\lim _{n \rightarrow \infty} & \left(\frac{\log n+\log (\log n)+\log \left(1+\frac{\log \log n}{\log n}\right)}{n}\right) \\
& =\lim _{n \rightarrow \infty}\left[\frac{1}{n}+\frac{1}{n \log n}+\frac{\frac{1}{n(\log n)^{2}}-\frac{\log (\log n)}{n(\log n)^{2}}}{1+\frac{\log (\log n)}{\log n}}\right] \rightarrow 0
\end{aligned}
$$

Clearly, both $n$ and $\log p_{n}$ for all $n \in \mathbb{N} \mid n \geq 1$ are positive divergent functions. Consequently, due to the fact that:

$$
\lim _{n \rightarrow \infty}\left(\frac{\log p_{n}}{n}\right) \rightarrow 0
$$

this of course implies $n \gg \log p_{n}$ for all $n \in \mathbb{N} \mid n \geq 6$. Table 2 demonstrates that $n>\log p_{n}$ for all $n \in \mathbb{N} \mid 1 \leq n \leq 6$. Consequently, $n \gg \log p_{n}$ for all $n \in \mathbb{N} \mid n \geq 1$. Now,

$$
\log \left(1+\frac{\mathcal{S U P}}{p_{n}}\right)=\log \left(p_{n}+\mathcal{S U P}\right)-\log \left(p_{n}\right)
$$

Which implies that Inequality 3.6,

$$
\frac{\log p_{n}}{n}>\log \left(1+\frac{\mathcal{S U P}}{p_{n}}\right) \equiv(n+1) \log p_{n}>n \log \left(p_{n}+\mathcal{S U P}\right)
$$

Suppose that the inequality 3.14 is false. First we exponentiate both sides of the inequality. Consequently, in accordance with the hypothesis for $p_{n} \geq 11$ :

$$
\left(p_{n}\right)^{(n+1)}-\left(p_{n}+\mathcal{S U P}\right)^{n}<0
$$

However, at $p_{5}=11$ the difference attains $\sim 1.13722 \times 10^{6}$ and diverges exponentially. Since the difference of terms is positive, we apply the Cauchy's Root Test:

$$
\lim _{n \rightarrow \infty} \sqrt[n]{\left|a_{n}\right|}=\lim _{n \rightarrow \infty} \sqrt[n]{\left(p_{n}\right)^{(n+1)}-\left(p_{n}+\mathcal{S U P}\right)^{n}} \rightarrow \infty
$$

Hence, the Cauchy's Root Test diverges, with the rate of divergence $\propto k p_{n} \mid k \sim 1$. This implies that a series formulated from the terms of the difference 3.15 diverges. Consequently, we have a contradiction to the initial hypothesis. Hence, it implies that:

$$
\left(p_{n}\right)^{(n+1)}>\left(p_{n}+\mathcal{S U P}\right)^{n} \quad \forall p_{n} \in \mathbb{N} \mid p_{n} \geq 11
$$


A straightforward computer calculation verifies that the relation 3.17 holds in the interval $2 \leq p_{n} \leq 11$. Now, since $p_{(n+1)}$ is defined as $p_{(n+1)}=p_{n}+\mathfrak{g}$, therefore, from the inequality $3.4,3.6$ and 3.17 we derive accordingly:

$$
\left(p_{n}\right)^{(n+1)}>\left(p_{(n+1)}\right)^{n} \quad \forall p_{n} \in \mathbb{N} \mid p_{n} \geq 2
$$

TABLE 2. Low range difference $n-\log p_{(n)}$

\begin{tabular}{|c|c|c|c|}
\hline$n-\log p_{(n)}$ & Difference value & $n-\log p_{(n)}$ & Difference value \\
\hline $1-\log 2$ & 0.306853 & $4-\log 7$ & 2.05409 \\
\hline $2-\log 3$ & 0.901388 & $5-\log 11$ & 2.6021 \\
\hline $3-\log 5$ & 1.39056 & $6-\log 13$ & 3.43505 \\
\hline
\end{tabular}

Remark 3.1. The functions implemented in the Theorem 3.1 are of the form:

$$
\sqrt[(n+k)]{p_{(n+k)}} \quad \forall k \in \mathbb{N} \cup\{0\}
$$

The property of this function, is in a way analogous to the property of the Supremum/Infimum bounds on $\log \mathcal{M}_{(t)}$; vide Remark 1.2. Keeping $n$ constant and allowing the variable $k$ to increase unboundedly, results in 3.18 being a decreasing function, converging asymptotically, strictly from above to 1. Firoozbakht's Theorem implements 3.18 with the variable $k=\{0,1\}$ respectively.

From the Firoozbakht Hypothesis $N^{\circ} 30$, the weak Firoozbakht's Maximal Prime Gaps Bound Lemma can be derived. Next, we may go one step further and define the strong Firoozbakht's Maximal Prime Gaps Bound. Both Lemmas and their proofs follow next.

Lemma 3.2 (The Weak Firoozbakht's Maximal Prime Gaps Bound).

For every $n \in \mathbb{N} \mid n \geq 11$ the maximal prime gaps satisfy the inequality:

$$
\mathfrak{g}=p_{(n+1)}-p_{n}<\left(\log p_{n}\right)^{2}-\log p_{n}=\mathcal{F} \mathcal{W}_{n}
$$

where $p_{(n)}$ is the $n$-th prime number.

Proof.

The weak form of the Firoozbakht's Maximal Prime Gaps Bound, for all $n \in$ $\mathbb{N} \mid n \geq 11$ asserts:

$$
\mathfrak{g}_{p_{n}}=p_{(n+1)}-p_{n}<\left(\log p_{n}\right)^{2}-\log p_{n}=\mathcal{F} \mathcal{W}_{n}
$$

The difference of the function $\mathcal{F} \mathcal{W}_{n}$ and the Supremum, defined by Theorem 2.6 is given by:

$$
\begin{aligned}
& {\left[\left(\left(\log p_{k}\right)^{2}-\log p_{k}\right)-\left(5\left(\log _{10} p_{k}\right)^{2}-\frac{15}{8}\left(\log _{10} p_{k}\right)\right)\right]=} \\
= & \left((\log 10)^{2}-5\right)\left(\log _{10} p_{k}\right)^{2}-\left((\log 10)-\frac{15}{8}\right)\left(\log _{10} p_{k}\right) \quad \forall p \in \mathbb{N} \mid p \geq 11
\end{aligned}
$$

The factors:

$$
(\log 10)^{2}-5 \sim 0.301898 \text { as well as }(\log 10)-\frac{15}{8} \sim 0.427585
$$


This implies that for all $p \in \mathbb{N} \mid p \geq 7$ the weak Firoozbakht's maximal prime gaps bound $\mathcal{F} \mathcal{W}_{n}$ lies above the Supremum, vide Table 4 in the Appendix,

$$
\left(\left(\log p_{k}\right)^{2}-\log p_{k}\right)>\left(5\left(\log _{10} p_{k}\right)^{2}-\frac{15}{8}\left(\log _{10} p_{k}\right)\right) \quad \forall p \in \mathbb{N} \mid p \geq 7
$$

Consequently by Theorem 2.6, the weak Firoozbakht's maximal prime gaps bound holds for all $p \in \mathbb{N} \mid p \geq 11$ :

$$
\mathfrak{g}=p_{(n+1)}-p_{n}<\left(\log p_{n}\right)^{2}-\log p_{n}
$$

Lemma 3.3 (The Strong Firoozbakht's Maximal Prime Gaps Bound).

For every $n \in \mathbb{N} \mid n \geq 37$ the maximal prime gaps satisfy the inequality:

$$
\mathfrak{g}=p_{(n+1)}-p_{n}<\left(\log p_{n}\right)^{2}-2 \log p_{n}+1=\mathcal{F} \mathcal{S}_{n}
$$

where $p_{(n)}$ is the $n$-th prime number.

Proof.

The strong form of the Firoozbakht's Maximal Prime Gaps Bound, for all $n \in$ $\mathbb{N} \mid n \geq 37$ asserts:

$$
\mathfrak{g}_{p_{n}}=p_{(n+1)}-p_{n}<\left(\log p_{n}\right)^{2}-2 \log p_{n}+1=\mathcal{F} \mathcal{S}_{n}
$$

The difference of $\mathcal{F} \mathcal{S}_{n}$ above and the Supremum defined by Theorem 2.6 is given by:

$$
\begin{aligned}
& \quad\left[\left(\left(\log p_{k}\right)^{2}-2 \log p_{k}+1\right)-\left(5\left(\log _{10} p_{k}\right)^{2}-\frac{15}{8}\left(\log _{10} p_{k}\right)\right)\right]= \\
& =\left((\log 10)^{2}-5\right)\left(\log _{10} p_{k}\right)^{2}-\left((2 \log 10)-\frac{15}{8}\right)\left(\log _{10} p_{k}\right) \quad \forall p \in \mathbb{N} \mid p \geq 11
\end{aligned}
$$

The factors:

$$
(\log 10)^{2}-5 \sim 0.301898 \text { as well as } 2(\log 10)-\frac{15}{8} \sim 2.73017
$$

Consequently, it is a matter of time before the leading quadratic term will begin to 'play the first violin'. The point where the difference 3.25 becomes positive, hence the point where the Supremum prime gaps bound intersects the stronger form of the Firoozbakht's prime gaps bound and remains below it, occurs:

$$
p_{k} \in \mathbb{N} \mid p_{k}>\exp \left(\frac{\sqrt{5\left(301(\log 10)^{2}-96(\log 10)^{3}\right)}+(16(\log 10)-15)(\log 10)}{2\left(8(\log 10)^{2}-40\right)}\right)
$$

Consequently, by Theorem 2.6, the stronger form of the Firoozbakht's maximal prime gaps bound holds for all $p \in \mathbb{N} \mid p>458034213$. For all $p \in \mathbb{N} \mid 37 \leq$ $p \leq 458034213$, a computer calculation verifies that the stronger form of the Firoozbakht's maximal prime gaps bound holds in this range, vide Table 3 below and Table 4 in the Appendix. Therefore the stronger form of the Firoozbakht maximal prime gaps bound holds for all $p \in \mathbb{N} \mid p \geq 37$. 
TABLE 3. Low range $\mathcal{F} \mathcal{S}_{n}$ vs primes within the range

\begin{tabular}{|c|c|c|c|c|c|}
\hline$n$ & $\mathcal{F S}_{n}$ & primes & $n$ & $\mathcal{F S}_{n}$ & primes \\
\hline 31 & 5.92429 & - & 61 & 9.67754 & 67 \\
\hline 37 & 6.81689 & 41,43 & 67 & 10.2701 & 71,73 \\
\hline 41 & 7.36347 & 43,47 & 71 & 10.6451 & 73,79 \\
\hline 43 & 7.62423 & 47 & 73 & 10.8271 & 79,83 \\
\hline 47 & 8.12334 & 53 & 79 & 11.3532 & 83,89 \\
\hline 53 & 8.82263 & 59,61 & 83 & 11.6885 & 89 \\
\hline 59 & 9.47124 & 61,67 & 89 & 12.1706 & 97,101 \\
\hline
\end{tabular}

Firoozbakht's Hypothesis is consistent with the Shank's Asymptotic Equality of Record Gaps Conjecture although it exposes a flaw and inconsistency in the Maier-Granville argument [14]:

$$
\mathfrak{g}_{p_{k}}=p_{(k+1)}-p_{k}<\mathcal{M}\left(\log p_{k}\right)^{2}
$$

with the limit as $k$ tends to infinity:

$$
\limsup _{k \rightarrow \infty}\left(\frac{p_{(k+1)}-p_{k}}{\left(\log p_{k}\right)^{2}}\right) \geq 2 \exp (-\gamma)
$$




\section{REFERENCES}

[1] R.J. Backlund, Über die Differenzen zwischen den Zahlen, die zu den ersten $n$ Primzahlen teilerfremd sind. Commentationes in honorem Ernesti Leonardi Lindelöf, Annales Acad. Sci. Fenn. 32 (1929), no. 2, 1-9.

[2] C. Bays and R.H. Hudson, A new bound for the smallest $x$ with $\pi_{x}>L i_{x}$, Mathematics Of Computation (1999).

[3] Enrico Bombieri, The shifting aspects of truth in mathematics, Institute For Advanced Study Princeton (2008).

[4] A. Brauer and H. Zeitz, Über eine zahlentheoretische Behauptung von Legendre, Sitzungsbericht, Berliner Math. Ges. 29 (1930), 116-125.

[5] Viggo Brun, Über das Goldbachsche Gesetz und die Anzahl der Primzahlpaare, Arch. Mat. Natur (1915).

[6] Chris K. Caldwell, The gaps between primes, 2006. http://primes.utm.edu/notes/gaps.html.

[7] Calvin C. Clawson, Mathematical mysteries; the beauty and magic of numbers, Plenum Press, New York, 1996.

[8] Harvey Cohn, Advanced number theory, Dover Publications, New York, 1980.

[9] Harald Cramer, On the order of magnitude of the difference between consecutive prime numbers, Acta Arithmetica (1936).

[10] R. Crandall and C. Pomerance, Prime numbers, a computational perspective, Springer Verlag, New York, 2005.

[11] Paul Erdös, On the difference of consecutive primes, Quarterly Journal Of Mathematics (1935).

[12] _ Some unsolved problems, Publications Of The Mathematical Institute Of The Hungarian Academy Of Sciences (1961).

[13] _ A survey of problems in combinatorial number theory, Annals Of Discrete Mathematics (1980).

[14] Andrew Granville, Harald Cramer and the distribution of prime numbers, Scandinavian Actuarial Journal (1995).

[15] G.H. Hardy and E.M. Wright, An introduction to the theory of numbers, Oxford University Press, London, 1968.

[16] Helge von Koch, Sur la distribution des nombres premiers, Acta Mathematica (1901).

[17] Edmund Landau, Vorlesungen über Zahlentheorie, Vol. II, S. Hirzel, Leipzig, 1927.

[18] H Maier, Primes in short intervals, Michigan Mathematical Journal (1985).

[19] Thomas R. Nicely, Some results of computational research in prime numbers (Computational number theory), 2009. http://www.trnicely.net.

[20] _ Skewes' problem, 2009. http://www.trnicely.net.

[21] János Pintz, Very large gaps between consecutive primes, Journal Of Number Theory (1997).

[22] S. Ramanujan, A proof of Bertrand's postulate, Journal Of Indian Mathematical Society 11 (1919), 181-182.

[23] Robert A. Rankin, The difference between consecutive prime numbers, Journal London Mathematical Society (1938).

[24] P. Ribenboim, The new book of prime number records, Springer Verlag, New York, 1996.

[25] Sebastian M. Ruiz, A result on prime numbers, Mathematical Gazette 81 (1997), no. 269, 269.

[26] Daniel Shanks, On maximal gaps between successive primes, Math. Comp. 18 (1964), 646651.

[27] Tomas Oliveira e Silva, Gaps between consecutive primes, 2006. www.ieeta.pt/ tos/gaps.html.

[28] K. Soundararajan, Small gaps between prime numbers: the work of Goldston-Pintz-Yildirim, Bulletin of the American Mathematical Society (2007).

[29] Eric W. Weisstein, Khinchin's constant, 1996. www.mathworld.wolfram.com/KhinchinsConstant.html.

[30] E. Westzynthius, Über die Differenzen Verteilung der Zahlen die zu den $n$ ersten Primzahlen teilerfremd sind, Comm. Phys. Math. Soc. Sci. Fenn. 5 (1931), 1-37. 


\section{Appendix}

4.1. Tabular data.

TABLE 4 . Theorem 2.6

\begin{tabular}{|c|c|c|c|c|}
\hline \multicolumn{5}{|c|}{ Maximal Prime Gaps } \\
\hline Gap start $p_{i}$ & $\begin{array}{l}\text { Actual } \\
\text { gap } \mathfrak{g}\end{array}$ & $\begin{array}{l}\text { Gap } \\
\text { estim. } \\
\mathcal{G} s_{\left(p_{i}\right)}\end{array}$ & $\begin{array}{l}\text { Firoozb. } \\
\text { Strong } \\
\text { Gap est. }\end{array}$ & $\begin{array}{l}\text { Firoozb. } \\
\text { Weak } \\
\text { Gap est. }\end{array}$ \\
\hline 7 & 3 & 1 & 0.894746 & 1.84066 \\
\hline 23 & 5 & 6 & 4.56034 & 6.69583 \\
\hline 89 & 7 & 15 & 12.1706 & 15.6592 \\
\hline 113 & 13 & 17 & 13.8934 & 17.6208 \\
\hline 523 & 17 & 31 & 27.6632 & 32.9228 \\
\hline 887 & 19 & 37 & 33.4991 & 39.287 \\
\hline 1129 & 21 & 40 & 36.3499 & 42.379 \\
\hline 1327 & 33 & 42 & 38.3245 & 44.5151 \\
\hline 9551 & 35 & 71 & 66.6574 & 74.8218 \\
\hline 15683 & 43 & 80 & 75.0014 & 83.6617 \\
\hline 19609 & 51 & 84 & 78.9209 & 87.8047 \\
\hline 31397 & 71 & 92 & 87.5061 & 96.8605 \\
\hline 155921 & 85 & 125 & 120.058 & 131.015 \\
\hline 360653 & 95 & 143 & 139.138 & 150.934 \\
\hline 370261 & 111 & 144 & 139.759 & 151.581 \\
\hline 492113 & 113 & 151 & 146.566 & 158.673 \\
\hline 1349533 & 117 & 176 & 172.01 & 185.126 \\
\hline 1357201 & 131 & 176 & 172.159 & 185.28 \\
\hline 2010733 & 147 & 186 & 182.628 & 196.142 \\
\hline 4652353 & 153 & 209 & 206.005 & 220.358 \\
\hline 17051707 & 179 & 247 & 244.978 & 260.629 \\
\hline 20831323 & 209 & 254 & 251.285 & 267.137 \\
\hline 47326693 & 219 & 280 & 277.975 & 294.648 \\
\hline 122164747 & 221 & 311 & 310.495 & 328.116 \\
\hline 189695659 & 233 & 327 & 326.197 & 344.258 \\
\hline 191912783 & 247 & 327 & 326.617 & 344.69 \\
\hline 387096133 & 249 & 352 & 352.47 & 371.244 \\
\hline 436273009 & 281 & 357 & 356.975 & 375.869 \\
\hline 1294268491 & 287 & 398 & 399.249 & 419.23 \\
\hline 1453168141 & 291 & 402 & 403.89 & 423.987 \\
\hline 2300942549 & 319 & 420 & 422.573 & 443.13 \\
\hline 3842610773 & 335 & 441 & 443.92 & 464.99 \\
\hline 4302407359 & 353 & 445 & 448.696 & 469.878 \\
\hline 10726904659 & 381 & 484 & 488.234 & 510.33 \\
\hline 20678048297 & 383 & 512 & 517.669 & 540.421 \\
\hline 22367084959 & 393 & 516 & 521.248 & 544.079 \\
\hline 25056082087 & 455 & 521 & 526.445 & 549.389 \\
\hline
\end{tabular}


TABLE 4. Continued

\begin{tabular}{|c|l|l|l|l|}
\hline \multicolumn{5}{|c|}{ Maximal Prime Gaps (Continued) } \\
\hline 42652618343 & 463 & 545 & 551.139 & 574.616 \\
\hline 127976334671 & 467 & 596 & 603.936 & 628.511 \\
\hline 182226896239 & 473 & 612 & 621.431 & 646.36 \\
\hline 241160624143 & 485 & 626 & 635.48 & 660.689 \\
\hline 297501075799 & 489 & 636 & 646.109 & 671.528 \\
\hline 303371455241 & 499 & 637 & 647.103 & 672.541 \\
\hline 304599508537 & 513 & 637 & 647.309 & 672.751 \\
\hline 416608695821 & 515 & 653 & 663.341 & 689.097 \\
\hline 461690510011 & 531 & 658 & 668.644 & 694.503 \\
\hline 614487453523 & 533 & 672 & 683.512 & 709.656 \\
\hline 738832927927 & 539 & 682 & 693.181 & 719.51 \\
\hline 1346294310749 & 581 & 712 & 725.137 & 752.066 \\
\hline 1408695493609 & 587 & 715 & 727.58 & 754.553 \\
\hline 1968188556461 & 601 & 732 & 745.734 & 773.042 \\
\hline 2614941710599 & 651 & 747 & 761.333 & 788.925 \\
\hline 7177162611713 & 673 & 802 & 818.07 & 846.672 \\
\hline 13829048559701 & 715 & 838 & 856.018 & 885.276 \\
\hline 19581334192423 & 765 & 858 & 876.491 & 906.097 \\
\hline 42842283925351 & 777 & 903 & 923.464 & 953.852 \\
\hline 90874329411493 & 803 & 948 & 969.731 & 1000.87 \\
\hline 171231342420521 & 805 & 986 & 1009.59 & 1041.36 \\
\hline 218209405436543 & 905 & 1001 & 1025.05 & 1057.07 \\
\hline 1189459969825483 & 915 & 1108 & 1136.52 & 1170.23 \\
\hline 1686994940955803 & 923 & 1130 & 1160.2 & 1194.26 \\
\hline 1693182318746371 & 1131 & 1131 & 1160.45 & 1194.52 \\
\hline 43841547845541059 & 1183 & 1353 & 1392.73 & 1430.05 \\
\hline 55350776431903243 & 1197 & 1370 & 1410.19 & 1447.74 \\
\hline 80873624627234849 & 1219 & 1397 & 1438.81 & 1476.74 \\
\hline 203986478517455989 & 1223 & 1465 & 1509.85 & 1548.71 \\
\hline 218034721194214273 & 1247 & 1470 & 1515.03 & 1553.96 \\
\hline 305405826521087869 & 1271 & 1495 & 1541.38 & 1580.64 \\
\hline 352521223451364323 & 1327 & 1506 & 1552.67 & 1592.07 \\
\hline 401429925999153707 & 1355 & 1516 & 1562.92 & 1602.46 \\
\hline 418032645936712127 & 1369 & 1519 & 1566.13 & 1605.7 \\
\hline 804212830686677669 & 1441 & 1569 & 1618.34 & 1658.57 \\
\hline 1425172824437699411 & 1475 & 1613 & 1664.71 & 1705.51 \\
\hline & & The End & & \\
\hline
\end{tabular}

